\title{
Characterization of the Differentiation and Leptin Secretion Profile of Adult Stem Cells on Patterned Polylactide Films
}

\author{
Aditya Chaubeya, Kevin J. Ross", Malcolm R. Leadbettero, \\ Cheryl T. Gomillion ${ }^{a}$ and Karen J. L. Burg ${ }^{a+}$ \\ Department of Bioengineering, 501 Rhodes Engineering Research Center, Clemson University, \\ Clemson, SC 29634, USA \\ b Department of Statistics \& Operations Research, 332 Hanes Hall, CB 3260, \\ University of North Carolina at Chapel Hill, Chapel Hill, NC 27599, USA
}

\begin{abstract}
Several issues need to be better understood before breast tissue engineering becomes a clinically viable option. One of the most important aspects is the interaction between cells and the microtopography of the implant surface. The aim of this study was to evaluate the efficacy of D1 cells, multipotent mouse bone marrow stromal precursors, in differentiating to adipocytes and to characterize their metabolic activity (lactic acid released and glucose consumed), leptin secretion and lipid production when cultured on patterned poly(L-lactide) (PLLA) films. It was determined that, by appropriate stimulation, the D1 cells displayed morphological characteristics of adipocytes and produced lipid. The results showed that a patterned surface did affect the rate of lipid production. Polynomial models were proposed to predict the amount of leptin secreted by the cells over a period of time.
\end{abstract}

Keywords

Adipocytes, D1 cells, leptin, lipid, microtexture, poly(L-lactide)

\section{Introduction}

The need for soft-tissue reconstruction or augmentation has increased continuously over the years. Breast cancer is the most common form of cancer affecting women [1]. The treatment options for breast cancer include lumpectomy, mastectomy and/or therapeutic treatments. Soft-tissue reconstruction is also crucial for the treatment of post-traumatic repair and congenital deformities. Current reconstruction procedures include implants, tissue flaps and tissue transplantation. Inherent problems exist with each option. The survival of grafted fat is low; generally graft-

\footnotetext{
* To whom correspondence should be addressed. E-mail: kburg@clemson.edu
} 
ing results in necrosis due to insufficient vascularization and results in resorption of the graft over time [2]. Additionally, the volume of fat available for grafting may be limited. Longevity and leaching of the constituent particles in synthetic replacement devices such as silicone implants is of the utmost concern, as is immune response to the bulk and degradant materials.

Tissue-engineering holds considerable promise as an alternative to existing options and provides a biologically-based solution. However, for any medical implant to be successful in the long-term, it must be successfully integrated into the body. Many strategies have been formulated to influence tissue-biomaterial interactions. The results of several studies detailed in the literature suggest that surfaces with microgrooves affect the orientation and migration of cells [3, 4]. Wan et al. [5] used microtopography to study the adhesion behavior of cells and found that cell adhesion was enhanced on poly(L-lactide) (PLLA) and polystyrene (PS) surfaces with nano-scale and micro-scale roughness compared to the smooth surfaces of the PLLA and PS. Parker et al. [6] evaluated tissue reaction around implants with different surface topographies and found that the application of microgrooves or random surface roughness to polymer implants did not have beneficial effects on peri-implant tissue healing in vivo. Thus, the role of microtopography in the healing response is not clear. Microtopography may, therefore, play an important role in guiding adipocyte function in a tissue-engineered device.

Adipocytes act as more than just energy reservoirs; they also exert significant regulatory influence on the brain in controlling body weight. The protein secreted by the adipocytes which plays a key role in this regulatory process is leptin. Leptin, discovered in 1994 by Zhang et al., is the product of the $o b$ gene coding for a 167-amino-acid protein and 21-amino-acid signal peptide [7] and acts via the hypothalamus region of the brain [8]. Leptin is also secreted by bone marrow adipocytes and stimulates myeloid and erythroid development [9].

Leptin provides the brain with information about the amount of fat present in the body and thereby acts as part of a feedback mechanism to control the amount of fat present. The majority of leptin circulates in the bound form in lean individuals and in the free form in obese subjects [10]. When the size and number of adipocytes increase, the cells produce leptin which is then released into plasma. Studies have demonstrated that plasma leptin concentration correlates with the amount of body fat present [11]. Insulin has been shown to increase the in vitro leptin production of rat and human adipocytes [12]. Leptin also counteracts insulin-mediated activation of glucose transport, glucose synthesis and lipogenesis in isolated rat adipocytes [13]. Both in vivo and in vitro studies in rodents and humans have shown that glucocorticoids enhance leptin gene transcription and leptin levels [14]. Leptin levels are also elevated in rats treated with dexamethasone [14]. Hence, the amount of leptin present in the cell culture medium was used in this study as an indicator to assess the functionality of the bone-marrow derived, differentiated adipocytes present on different PLLA films. 
This proof-of-concept study evaluated the effect of surface topography on the differentiation of D1 cells, evaluating changes in lipid production and leptin levels in response to topographical feature changes. We propose that by controlling the level of cellular differentiation in a cell-based device, one can more efficiently control the device's fate in vivo. Thus, the bone marrow-derived mesenchymal stem cells used in this study provide an example of a ready source of proliferating cells whose timing and level of differentiation can be controlled by design.

\section{Materials and Methods}

\subsection{Substrate Fabrication}

Photolithography was employed (Microelectronics Division, Department of Electrical and Computer Engineering, Clemson University) to etch micro-grooved patterns on a silicon wafer. Various other textures were evaluated before selecting this particular pattern, which allows easy, highly repeatable manufacturing. The manufactured wafer had horizontal surface grooves of $3 \mu \mathrm{m}$ width over its surface and $100 \mu \mathrm{m}$ separation (pitch). The depth of each groove was $1.5 \mu \mathrm{m}$ and the diameter of the wafer was $7.6 \mathrm{~cm}$. These dimensions were selected to provide a surface texture sufficient to allow a large fraction of fully differentiated adipocytes to span grooves, thus ensuring cellular interaction with the texture. The wafer was used as a template for patterned film fabrication.

Polymer films were solvent-cast, using a silicon wafer template. Specifically, $1 \mathrm{~g}$ PLLA (Boehringer Ingelheim, molecular weight $140 \mathrm{~kg} / \mathrm{mol}$ ) was mixed with $10 \mathrm{ml}$ dichloromethane (VWR) to make a $10 \%$ polymer solution. The template was then covered with $10 \mathrm{ml}$ of the polymer solution. The solvent was allowed to evaporate slowly and, after the solvent evaporation was complete, the PLLA film was removed from the substrate. Unpatterned, control PLLA films were similarly made using a Petri dish as a template; select specimens were examined by scanning electron microscope (SEM). Round discs (2 cm diameter) were punched from both the patterned and the plain films. These discs were placed in tissue-culture-treated multiwell plates (Corning) which were coated with agarose (Sigma) to prevent the cells from preferentially adhering to the well surface. A Teflon ring (Fluoro-Plastics) was placed on each disc to prevent disc flotation. The tissue-culture-treated polystyrene (PS) surface in the multi-well plate was used to demonstrate viability and proliferation of the cells in standard culture. The textured PLLA control was cast from a glass Petri dish, thus eliminating the potential for dissolution of the mold and subsequent contamination of the films by leachables. These samples were used simply to demonstrate that the addition of surface texture (in the absence of substrate chemical differences) would cause differences in cellular differentiation. For consistency, each control well was also fitted with a Teflon ring. The multi-well plates with discs and Teflon rings were sterilized using ethylene oxide (Andersen Sterilizers). 


\subsection{Cell Culture Conditions}

All parameters chosen for this study were based on previous experiments, conducted to ensure that confluence was achieved in all systems in a similar time frame. D1 cells (ATCC) of passage 29 were seeded on all the surfaces (textured films, plain films and PS surfaces) at a density of $3.4 \times 10^{4}$ cells/well (20\% confluence). The cells were maintained in Dulbecco's Modified Eagle Medium (DMEM; ATCC) supplemented with fetal bovine serum (Mediatech), fungizone (Invitrogen) and antibiotic-antimicotic (Invitrogen); the supplemented medium hereafter will be referred to as DMEM-C. Cells were incubated at $37^{\circ} \mathrm{C}$ and $5 \% \mathrm{CO}_{2}$. The cells reached confluence in two days under these conditions; adipogenesis was induced by adding a differentiation induction cocktail of $0.5 \mu \mathrm{M}$ dexamethasone (Sigma), $1 \mu \mathrm{g} / \mathrm{ml}$ insulin (Sigma) and $0.5 \mathrm{mM}$ 1-methyl-3-isobutylmethylxanthene (IBMX, Sigma) to DMEM-C.

Eight 12-well plates were used in this experiment, seven for histological staining using Oil Red $\mathrm{O}$ and one for measuring lactic acid released/glucose consumed and leptin released by the cells. Each well plate contained six samples, one per well, including three PLLA patterned films and three plain PLLA films. Three additional wells per plate served as polystyrene controls.

\subsection{Oil Red O Staining}

To determine the increase in the percent area covered by lipid over a two week span, Oil Red $\mathrm{O}$ was used to stain the cytoplasmic lipid droplets. This analysis was performed at seven different time-points, i.e., on days 2, 4, 6, 8, 10, 12 and 14 . Image Pro ${ }^{\circledR}$ image analysis software was used to quantify the amount of lipid. Six images of each sample surface were taken at each time-point using Image Pro® and a Zeiss inverted microscope. A total of 18 images per surface-type per time-point were taken. All images were taken at a total magnification of $320 \mathrm{x}$. One well-plate was used for each individual time-point. Each well-plate contained six samples, one per well, including three PLLA patterned films and three plain PLLA films. Three additional wells per plate served as polystyrene controls. All the surfaces in a well-plate were stained with Oil Red O. The resulting red staining of cytoplasmic lipid was used to identify differentiated cells. The total area of the stained lipid

was measured using Image Pro ${ }^{\circledR}$. The total area represents the total cell population, because the cells were confluent in monolayer.

\subsection{Lactic Acid/Glucose Analysis}

Medium $(100 \mu \mathrm{l})$ was removed from each of the experimental and control wells every day, and the medium was changed every other day. The amounts of lactic acid and glucose present in the extracted culture medium were subsequently measured using a biochemistry analyzer (YSI). 


\subsection{Leptin Measurement}

The leptin ELISA was performed according to the manufacturer's instructions (R\&D Systems). Medium was isolated daily from all the samples and the controls and stored (at $-20^{\circ} \mathrm{C}$ ) until the end of the experiment, after which the ELISA was performed. Values are expressed as pg leptin/construct (scaffold). Aliquots of medium from undifferentiated cells on each surface were used as the leptin ELISA control samples for the corresponding surfaces.

\subsection{Statistical Analysis}

Standard statistical analyses were performed to compare amounts of lipid formation on the three materials, and confidence intervals were provided for their pairwise differences, at each of the time-points for which Oil Red O staining was conducted. Leptin secretion was modeled by appropriate regression analysis, and differences between these measurements for the three scaffold materials were tested by analysis of variance methods. Detailed methodology will be described subsequently.

\section{Results}

Scanning electron microscopy (SEM) was used to image the surface of the patterned and plain PLLA films following fabrication. Representative images of the surfaces and cells cultured on the surfaces are shown in Fig. 1. Upon viewing samples under higher magnification, lipid droplets are seen accumulating within the cells. Oil Red O staining was performed on cells seeded on the patterned and plain PLLA films and on the PS surface over seven different time-points (days 2, 4, 6, 8, 10, 12 and 14) in order to quantify the increase in the percent area occupied by lipid droplets over the two-week period on all the surfaces.

Statistical analysis revealed an overall significant difference in the lipid accumulated between the three scaffold types. Also, the amount of lipid accumulated by the cells on plain and patterned films was significantly different on days 6, 8, 10, 12 and 14 ( $\mathrm{P}<0.01)$. Figure 2 shows the percent area occupied by the lipid droplets on all the surfaces over the period of two weeks. The cells on the patterned surface accumulated the highest amount of lipid and the cells on the plain PLLA surface had the lowest amount of lipid.

\subsection{Lactic Acid Released and Glucose Consumed}

Lactic acid is a product of cellular anaerobic metabolism, and glucose is the primary energy source for cellular metabolism. Metabolic activity was assessed to investigate if indeed any surface-induced differences existed. No significant difference $(P>0.05)$ was found between the amounts of lactic acid released by the cells on any of the surfaces (Fig. 3) or between the amounts of glucose consumed by the cells on any of the surfaces (Fig. 4). 


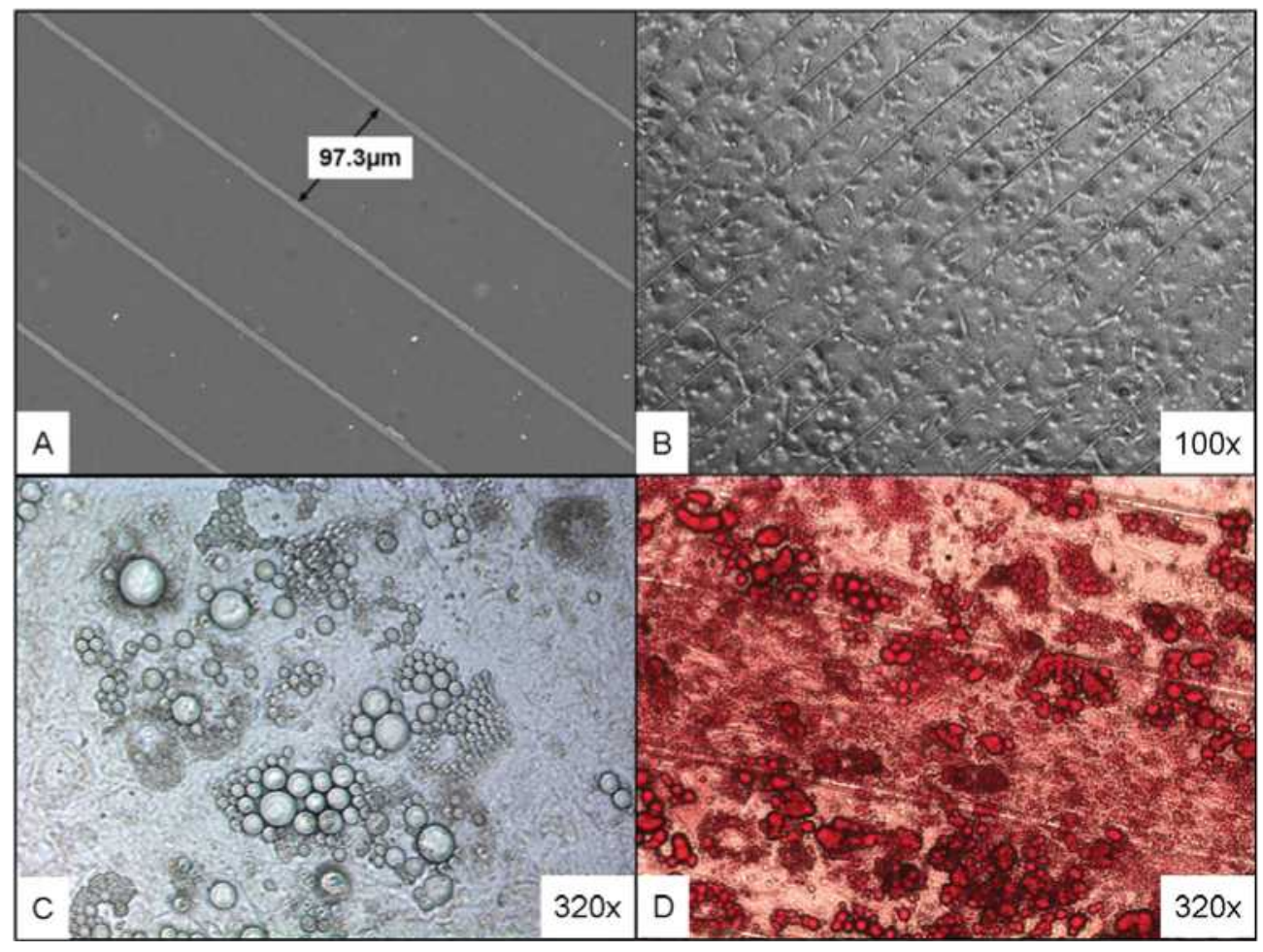

$\beta$ Figure 1. (A) Representative SEM image displaying the surface of a patterned PLLA film. The measured pitch between the microgrooves was approx. $97 \mu \mathrm{m}$. (B) D1 cells attached to patterned PLLA film and proliferating on the surface following 1 day of culture. (C) Lipid droplets accumulated within differentiated cells following 21 days of culture. (D) Lipid droplets stained with Oil Red O following culture on a patterned PLLA film for 21 days. Magnifications on images represent total magnification. This figure is published in colour in the online edition that can be accessed via http://www.brill.nl/jbs

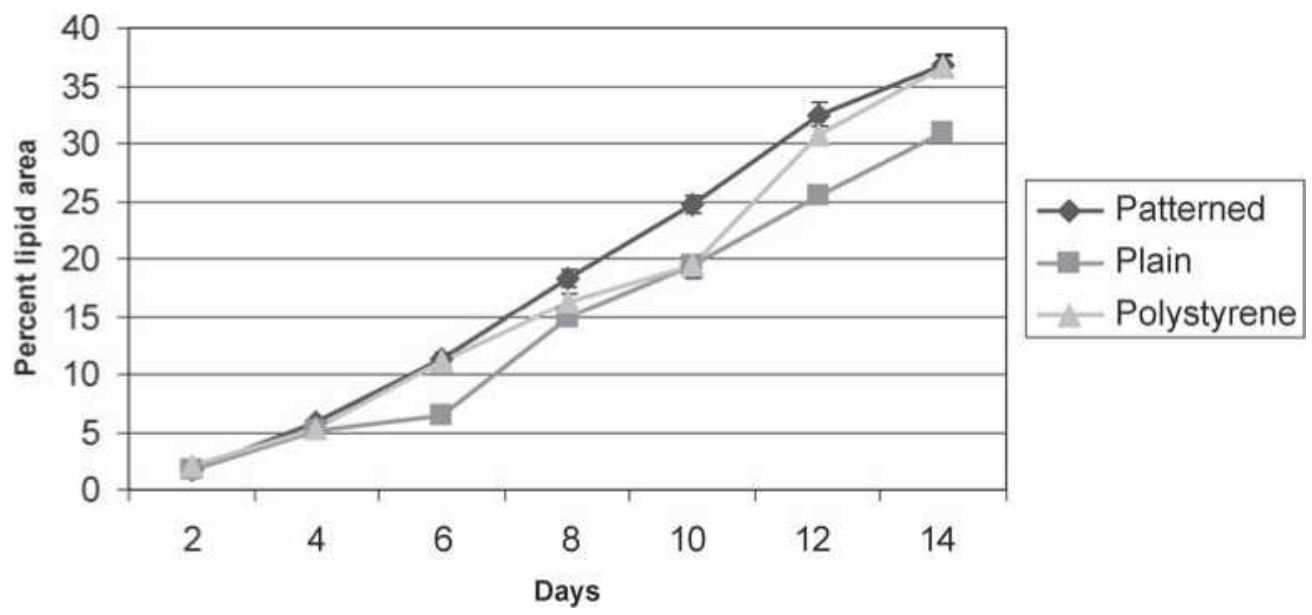

Figure 2. Percent area occupied by lipid on all the three surfaces. Each data point represents a mean of three values and the error bars denote standard error of the mean (SEM).

\subsection{Leptin Release}

The amount of leptin released from D1s was measured over a period of twenty days. Measurement of leptin concentration in the culture medium of the constructs 


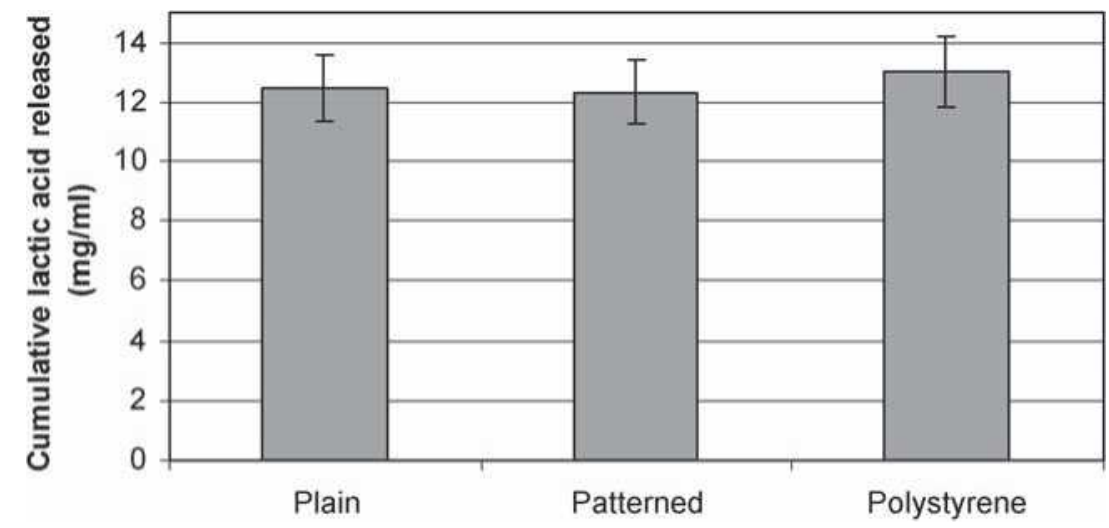

Figure 3. Cumulative lactic acid released by the cells on the different surfaces over the 14-day study. Error bars denote SEM.

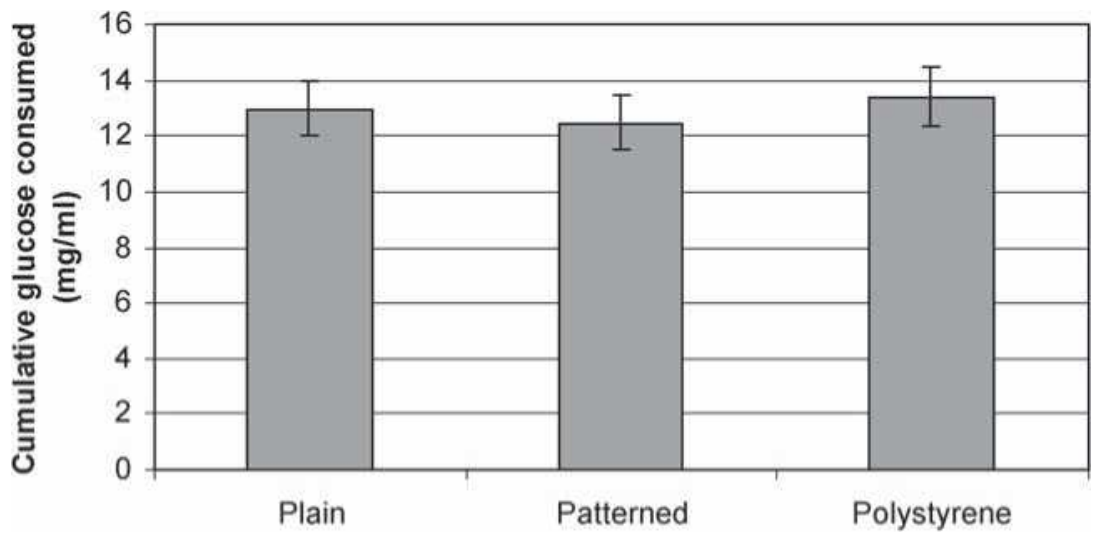

Figure 4. Cumulative glucose consumed by the cells on different surfaces over the 14-day study. Error bars denote SEM.

revealed that the cells on the polystyrene surface released significantly higher amounts of leptin $(P<0.05)$ as compared to cells on the patterned and plain surfaces; however, no significant difference was found in the leptin released by the cells on plain surfaces versus leptin released by those on patterned surfaces. The amount of leptin released by the D1 cells was $3.92 \mathrm{ng}$ leptin/plain film, $4.07 \mathrm{ng}$ leptin/patterned film and $4.56 \mathrm{ng}$ leptin/polystyrene surface, respectively. Figure 5 shows the progression of daily leptin values with time.

Figure 5 shows the leptin released by the cells on the different surfaces. Cells on the PS scaffolds generally exhibit the highest level of leptin secretion, while the levels for cells on the Plain and Patterned scaffolds are similar. Cells on the PS scaffolds also exhibit a somewhat higher level of variability than those on the other two scaffolds. Leptin secretion increases linearly over time.

Analysis of covariance was performed to test for differences in the time trend due to the scaffold. Table 1 summarizes the results. After accounting for a linear time trend, a significant difference in the overall mean leptin values across the different scaffolds exists, which can be incorporated in the model by adjusting the intercept of the linear trend. However, after accounting for the difference 


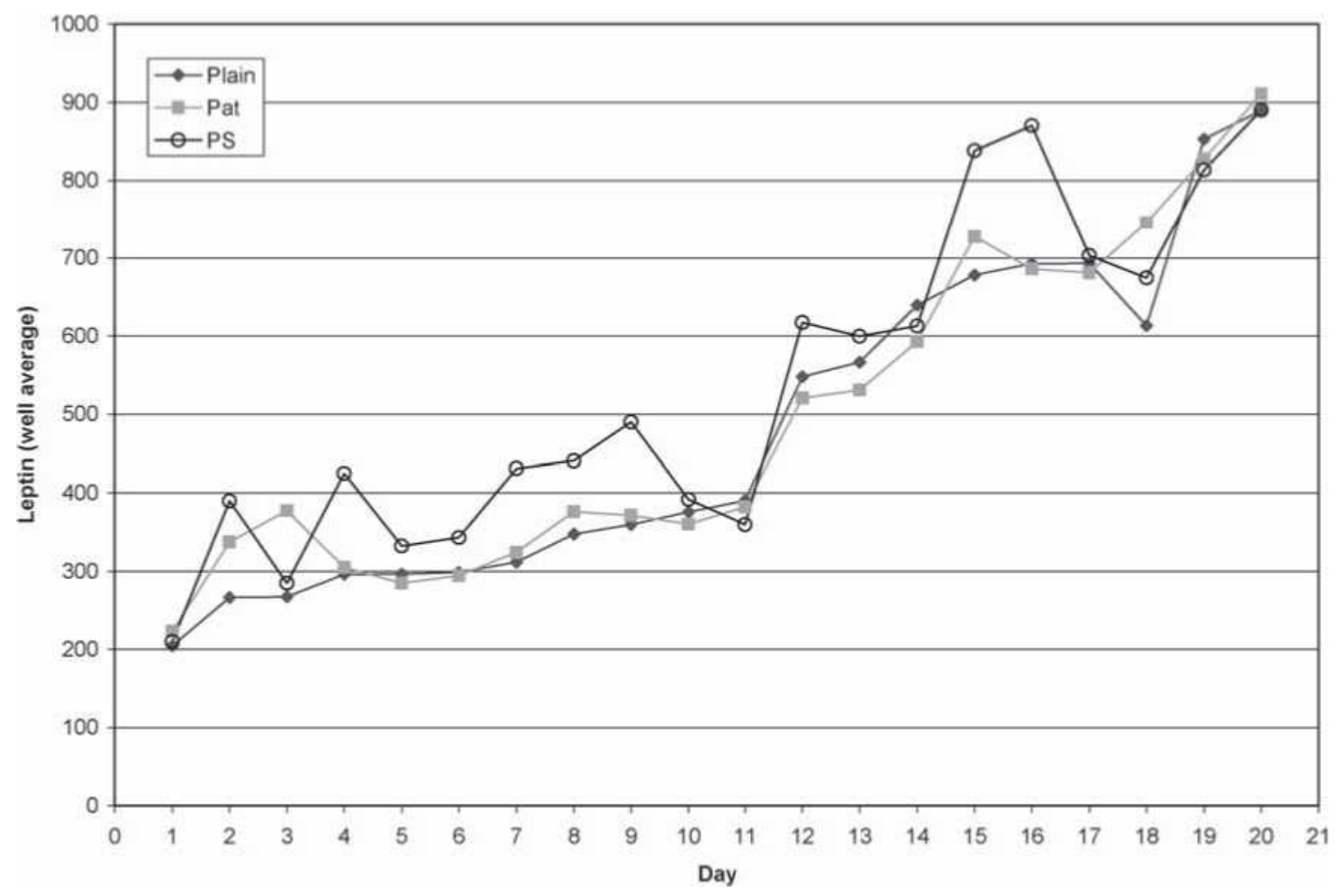

Figure 5. Leptin concentration in the three culture conditions over time.

Table 1.

ANOVA: differences in time trend for released leptin due to the scaffold type

\begin{tabular}{llrr}
\hline Source & Degrees of freedom & Sum of squares & $P$ \\
\hline Day & 1 & 6422510 & $<0.0001$ \\
Scaffold & 2 & 104194 & 0.0002 \\
Day x scaffold & 2 & 4273 & 0.6896 \\
\hline
\end{tabular}

Table 2.

Linear time trend for leptin released

\begin{tabular}{lccc} 
& Parameter estimate & SE & $P$ \\
\hline Intercept & 125.05 & 10.18 & $<0.0001$ \\
Patterned & -8.88 & 10.09 & 0.3802 \\
Plain & 0.00 & N/A & N/A \\
PS & 19.36 & 10.39 & 0.0644 \\
Day & 34.99 & 0.73 & $<0.0001$ \\
\hline
\end{tabular}

in mean due to scaffold, allowing for the different slopes in the time trend does not significantly contribute to the model. Table 2 summarizes the parameter estimates. 
Table 3.

Outlier values for leptin released for the different scaffolds

\begin{tabular}{ll}
\hline Scaffold & Observations removed (days) \\
\hline Plain & 11,18 \\
Patterned & $2,3,11$ \\
PS & $2,4,11,15,16$ \\
\hline
\end{tabular}

Table 4.

ANOVA for leptin released after discounting the outlier values

\begin{tabular}{llcr}
\hline Source & Degrees of freedom & Sum of squares & $P$ \\
\hline Day & 1 & 6012738 & $<0.0001$ \\
Scaffold & 2 & 9856 & 0.0241 \\
Day x scaffold & 2 & 7351 & 0.0609 \\
\hline
\end{tabular}

To estimate the parameters uniquely, the coefficient for the plain scaffold was set to 0. The coefficients for patterned and PS represent the adjustment to the intercept of the trend from the baseline plain values. Note that the coefficient for the patterned scaffold is not statistically significant; that is, there is no significant difference between the intercept of the linear trend of the plain and that of the patterned scaffolds. These parameter estimates translate into the following model:

$$
\begin{aligned}
& \text { Plain: } \quad \text { Leptin secreted (day) }=125.05+34.99 \text { day; } \\
& \text { Patterned: } \text { Leptin secreted (day) }=116.17+34.99 \text { day; } \\
& \text { PS: } \quad \text { Leptin secreted (day) }=144.41+34.99 \text { day. }
\end{aligned}
$$

The above model is based on all data observations. However, several observations (see Table 3) were identified as either outliers or influential contributors in this analysis. The analysis of covariance was performed again with these observations excluded and Table 4 summarizes the results. There is still a significant contribution due to the overall mean for each scaffold. Yet now there is also some evidence of a significant difference in the slope of the time trend due to differences across the scaffold. Inclusion or exclusion of the observations in Table 3 significantly affects the resulting model. Table 5 summarizes the parameter estimates for the revised model.

Again, the coefficients for the plain scaffold were set to 0 . The coefficients for patterned and PS above represent the adjustments to the slope and intercept from the baseline plain values. Note that the coefficients for the patterned scaffold are not statistically significant and, thus, there is no significant difference in the linear trend (slope and intercept) between the plain and patterned scaffolds. These parameter 
Table 5.

Linear time trend for leptin released after discounting the outlier values

\begin{tabular}{lccc}
\hline & Parameter estimate & SE & $P$ \\
\hline Intercept & 118.54 & 13.83 & $<0.0001$ \\
Patterned & -21.39 & 21.36 & 0.3181 \\
Plain & 0 & N/A & N/A \\
PS & 53.22 & 21.20 & 0.0132 \\
Day & 35.64 & 1.19 & $<0.0001$ \\
Day x patterned & 1.02 & 1.75 & 0.5612 \\
Day x plain & 0 & N/A & N/A \\
Day x PS & -3.18 & 1.77 & 0.0750 \\
& & & \\
\hline
\end{tabular}

estimates translate into the following model:

$$
\begin{array}{ll}
\text { Plain: } & \text { Leptin secreted }(\text { day })=118.54+35.64 \text { day; } \\
\text { Patterned: } & \text { Leptin secreted }(\text { day })=97.14+36.66 \text { day; } \\
\text { PS: } & \text { Leptin secreted }(\text { day }=171.76+32.46 \text { day }
\end{array}
$$

Figure 6 displays the estimated model along with the daily mean leptin values. Figure 7 displays the corresponding residuals. The main feature of the residual plot is the 'within day' correlation between the residuals. Figure 8 shows the cumulative amount of leptin released for all scaffolds.

\section{Discussion}

The aim of this study was to examine the differentiation of D1 cells into adipocytes and to assess their functionality, i.e., the cellular response, in terms of lipid production, leptin secretion and metabolism (lactic acid released and glucose consumed) when cultured on PLLA films with defined surface topographies. Surface topography considerably affects the proliferation and differentiation of cells and, hence, microtopography is a useful tool to better understand the cell-material interaction in a carefully designed system. The size of an adipocyte typically ranges from around 20 pm to 120 pm; hence, grooves which were separated by 100 pm were assessed.

Oil Red $\mathrm{O}$ staining was conducted to determine the amount of lipid accumulated by the cells and the trend of lipid production by the cells on the different surfaces over a two week period. The D1 cells accumulated lipid droplets on all surfaces, the amount of which increased significantly over the period of 2 weeks. The area covered by lipid was significantly different on each surface. The results indicated that the patterned surface contributed to the differentiation of D1s, as the cells cultured on the patterned surface accumulated significantly higher amounts of lipid from day 6 to the end of the last time-point (day 14). No difference in accumulated lipid was noted on days 2 and 4, which is not surprising as the cells do not start differentiating until after a few days of treatment with the cocktail. Also, a more linear 


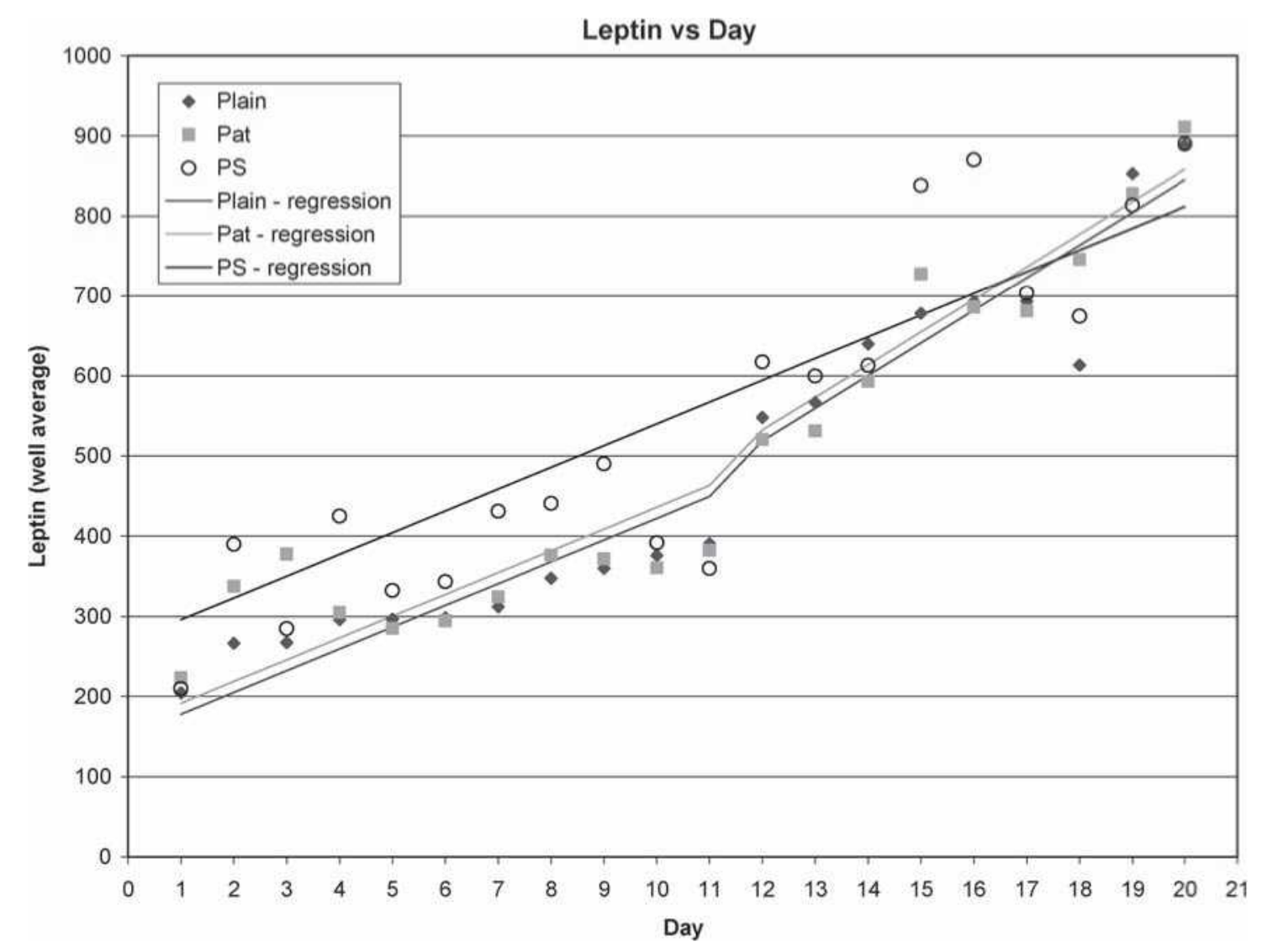

Figure 6. Linear trend of leptin released, based on the model proposed.

trend of lipid accumulation by the cells cultured on patterned PLLA films was observed throughout the period of the study, as compared to the cells cultured on the other two surfaces. This trend might be attributed to a more controlled and gradual differentiation (and, hence, lipid accumulation) due to the surface microtexture.

Studies in the literature [4, 15] suggest that microgrooves influence cell alignment, where two critical factors are groove width and depth. Clark et al. [16] have shown that cell alignment is significantly affected by differences in groove depth, but that differences in groove width are not very critical in influencing cell alignment. There is no clear consensus regarding the optimum groove width or depth, as this value depends on several factors, such as the cell-type or the in vitro conditions of the experiments conducted. Results from preliminary studies in our laboratory have shown that groove width does affect the rate of differentiation of D1s to adipocytes. In the current experiment, however, one combination of groove width and depth was used; further studies with various groove sizes are needed to better understand and quantify the significance of these parameters. Many studies have employed microtopography as a tool to study alignment of cells of osteoblastic lineage [17, 18]; however, there are very few studies that investigate the effect of microtopography on soft-tissue differentiation and functionality. In this study a hormone differentiation inducer was used in all cases; thus, differences in cellular 


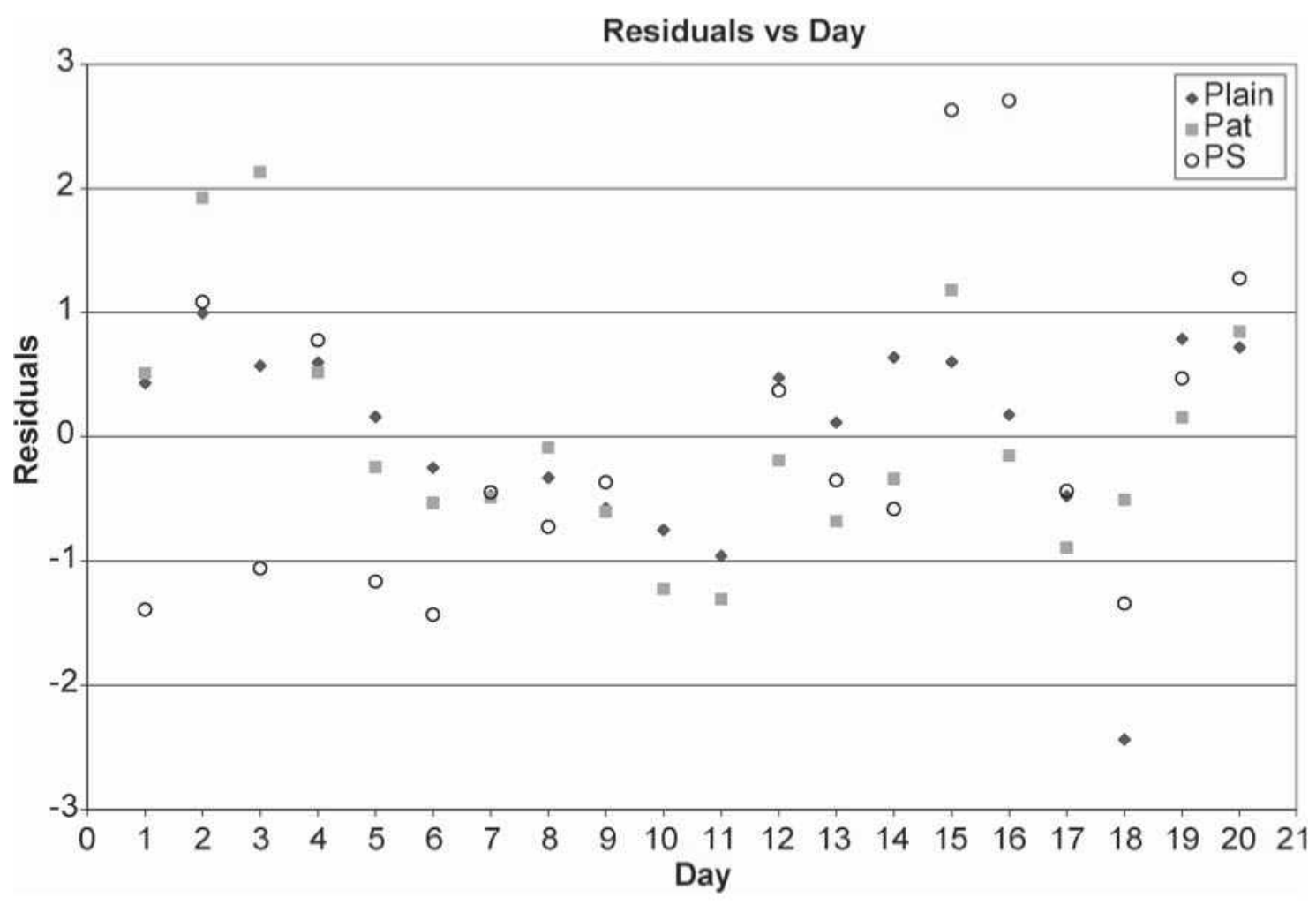

Figure 7. Residuals for the linear trend of the leptin released.

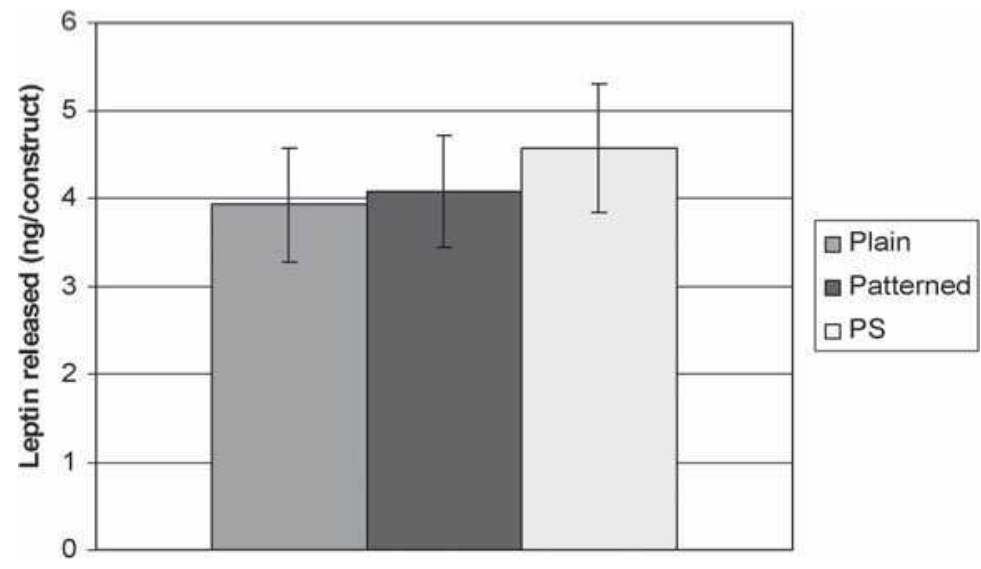

Figure 8. Cumulative leptin released by D1 cells on different surfaces. Error bars denote standard error of mean (SEM).

behavior were attributed to surface microtopography. However, the role of the substrate and its surface texture on the differentiation of cells also needs further study. It has been reported [19] that bone cell differentiation and proliferation is affected by the interaction between hormones, growth factors and surface microtopgraphy.

A very linear trend (and significantly higher amount of lipid) was observed in the lipid accumulation by the cells on patterned PLLA films as compared with cells cultured on other surfaces. As all the parameters were same for the cells seeded 
on the three surfaces, it can be inferred that the patterning of the PLLA films does have an effect on the cellular behavior. Although the results indicate that this particular patterned surface is capable of influencing the differentiation, further work must be completed to pinpoint the exact mechanism by which this linear trend is orchestrated.

Cellular metabolism, as a function of lactic acid released and glucose consumed, was also analyzed. There was no significant difference in either of the parameters between the cells cultured on PLLA films and those on tissue-culture-treated polystyrene surface, suggesting that PLLA can serve as a good scaffold material. However, literature reports [20] state that cells are more proliferative on a natural material like gelatin than PLLA. Studies in the literature have focused on adipocytes seeded on gelatin microcarriers; hence, the findings could be due to differences between surface chemistry or roughness. Another reason for the difference in results could be due to the fact that cells cultured on three-dimensional scaffolds (beads) tend to proliferate and differentiate differently than on two-dimensional surfaces (films). Further investigation is required to better quantify and compare cell behavior on natural and synthetic polymers.

In the past decade, since its discovery in 1994, leptin has received tremendous attention in the field of obesity research. Leptin is a key protein secreted by adipocytes and is also a marker used to assess the functionality of adipocytes. To the best of our knowledge, this study is the first to characterize the leptin secretion profile of D1s that have differentiated into adipocytes. The results show that D1s cultured on all the surfaces visually display the adipocyte phenotype. Several studies have examined the process of adult stem cell commitment and differentiation to osteogenic lineage; however, much less is known about the conditions favoring adipogenic differentiation. The results further highlight the adipogenic potential of multipotent mouse bone marrow stromal precursor (D1) cells. The cells seeded on tissue-culture-treated polystyrene surface released the highest amount of leptin into the culture medium as compared to the cells seeded on the polymer films. This result suggests that PLLA has an inhibitory influence on the leptin secretion ability of D1s. More work is needed to better elucidate the leptin secretion ability of these cells on different polymeric materials (for example, synthetic versus natural) and to determine whether or not the leptin release values cited in Table 3 are outliers and, if so, the cause for the outlying behavior, i.e., chance variation versus experimental error.

Once the cells became confluent, they were consistently maintained in the adipogenic cocktail; however, results from the literature [21] suggest that extended 'resting periods' in the absence of adipogenesis inducers (after the onset of differentiation) enhances the differentiation process and increases the leptin mRNA levels of human mesenchymal stem cells. It is of interest to investigate if there is a similar pattern observed in D1 cells. Two key transcription factors in adipocyte differentiation, $\operatorname{PPAR} \gamma$ and $\mathrm{C} / \mathrm{EBP} \alpha$, affect leptin gene expression in opposite ways. While PPAR $\gamma$ decreases leptin expression [21-23], C/EBP $\alpha$ induces leptin gene 
expression [21-26]. The presence of multiple mechanisms suggests that the level of circulating leptin is controlled by a complex set of signals. Also, cAMP has been shown to decrease the leptin level in rodents [27]. A hormonal cocktail, including IBMX, was employed to induce differentiation. IBMX increases the production of cAMP and, hence, should serve to decrease the production of leptin. Leptin has also been implicated in regulating body weight, in fetomaternal signaling [28] and in hematopoiesis [29].

In a recent study it was shown that physiological concentrations of leptin (25-100 ng/ml) can stimulate the proliferation of both normal and malignant breast epithelial cells [30]. Leptin enhanced anchorage-independent growth of select breast cancer cell lines but did not similarly enhance the growth of normal cell lines. It has also been shown that leptin might play a role in the development of mammary tumors [31]. Elevated serum protein levels associated with obesity may also promote breast cancer development. To better design tissue-engineered constructs for breast reconstruction, it will be necessary to address more fully the role of leptin, which is a key regulatory adipocyte protein.

Preadipocytes comprise the standard, current model system for studying adipogenesis. However, the inherent disadvantages posed, such as the limited number of cells that can be obtained from an individual and limited life cycle, necessitate further research with marrow stromal cells, which do not have these limitations. There is a growing interest in natural polymers as well as the evaluation of D1 cell behavior on natural polymers like collagen. Our study offers a functional characterization of D1 cells and investigates their behavior (differentiation and metabolism) when cultured on polymers with a defined topography. This study offers a starting platform toward a better understanding of the interaction between synthetic scaffold and the cellular component of a tissue-engineered device.

\section{Conclusions}

Patterned PLLA films were used to compare the behavior of D1 cells on plain PLLA films and tissue-culture-treated PS surface by assessment of lipid production, metabolism and leptin secretion. Lipid accumulation was significantly different for cells on the patterned scaffolds when compared to plain scaffolds. No differences were detected in the metabolism of the cells on any of the scaffolds, as measured by lactic acid released and glucose consumed. Polynomial models were proposed to predict the amount of leptin released by the cells as a function of time.

\section{References}

1. B. E. Henderson, R. K. Ross and M. C. Pike, Science 259, 633 (1993).

2. C. W. Patrick Jr., P. B. Chauvin and G. L. Robb, in: Frontiers in Tissue Engineering, C. W. Patrick Jr., A. G. Mikos and L. V. Mclntire (Eds), p. 369. Elsevier Science, Oxford (1998).

3. A. F. von Recum and T. G. van Kooten, J. Biomater. Sci. Polymer Edn 7, 181 (1995).

4. J. A. Schmidt and A. F. von Recum, Biomaterials 13, 1059 (1992). 
5. Y. Wan, Y. Wang, Z. Liu, X. Qu, B. Han, J. Bei and S. Wang, Biomaterials 26, 4453 (2005).

6. J. A. Parker, X. F. Walboomers, J. W. Von den Hoff, J. C. Maltha and J. A. Jansen, J. Biomed. Mater. Res. 61, 91 (2002).

7. Y. Zhang, R. Proenca, M. Maffei, M. Barone, L. Leopold and J. M. Friedman, Nature 372, 425 (1994).

8. N. Satoh, Y. Ogawa, G. Katsuura, T. Ttsuji, H. Masuzaki, J. Hiraoka, T. Okazaki, M. Tamaki, M. Hayase, Y. Yohimasa, S. Nishi, K. Hosada and K. Nakao, Endocrinology 138, 947 (1997).

9. M. Konopleva, A. Mikhail, Z. Estrov, S. Zhao, D. Harris, G. Sanchez-Williams, S. M. Kornblau, J. Dong, K. O. Kliche, S. Jiang, H. R. Snodgrass, E. H. Estey and M. Andreef, Blood 93, 1668 (1999) .

10. M. K. Sinha, I. Opentanova, J. P. Ohannesian, J. W. Kolaczynski, M. L. Heiman, J. Hale, G. W. Becker, R. R. Bowsher, T. W. Stephens and J. F. Karo, J. Clin. Invest. 98, 1277 (1996).

11. R. V. Considine, M. K. Sinha, M. L. Heiman, A. Kriauciunas, T. W. Stephens, M. R. Nyce, J. P. Ohannesian, C. C. Marco, L. J. McKee and T. L. Bauer, N. Engl. J. Med. 334, 292 (1996).

12. J. Rentsch andM. Chiesi, FEBS Lett. 379, 55 (1996).

13. G. Muller, J. Ertl, M. Gerl and G. Preibisch, J. Biol. Chem. 272, 10585 (1997).

14. P. De Vos, R. Saladin, J. Auwerx and B. Staels, J. Biol. Chem. 270, 15958 (1995).

15. K. Matsuzaka, F. Walboomers, A. De Ruijter and J. A. Jansen, Clin. Oral Implant. Res. 11, 325 (2000) .

16. P. Clark, P. Connolly, A. S. Curtis, J. A. Dow and C. D. Wilkinson, Development 108, 635 (1990).

17. B. D. Boyan, L. F. Bonewald, E. P. Paschalis, C. H. Lohmann, J. Rosser, D. L. Cochran, D. D. Dean, Z. Z. Schwartz and A. L. Boskey, Calcif. Tissue Int. 71, 519 (2002).

18. S. Lossdorfer, Z. Schwartz, L. Wang, C. H. Lohmann, J. D. Turner, M. Weiland, D. L. Cochran and B. D. Boyan, J. Biomed. Mater. Res. A 70, 361 (2004).

19. J. Y. Martin, Z. Schwartz, T. W. Hummert, D. M. Schraub, J. Simpson, J. Lankford Jr., D. D. Dean, D. L. Cochran and B. D. Boyan, J. Biomed. Mater. Res. 29, 389 (1995).

20. N. Cavin, MS Thesis. Bioengineering Department, Clemson University, Clemson, SC (2005).

21. B. Zhang, J. Berger, E. Hu, D. Szalkowski, S. White-Carrington, B. M. Spiegelman and D. E. Moller, Mol. Endocrinol. 10,1457 (1996).

22. C. B. Kallen and M. A. Lazar, Proc. Natl. Acad. Sci. USA 93, 5793 (1996).

23. P. De Vos, A. M. Lefebvre, S. G. Miller, M. Guerre-Millo, K. Wong, R. Saladin, L. G. Hamann, B. Staels, M. R. Briggs and J. Auwerx, J. Clin. Invest. 98,1004 (1996).

24. Y. He, H. Chen, M. J. Quon and M. Reitman, J. Biol. Chem. 270, 28887 (1995).

25. A. N. Hollenberg, V. S. Susulic, J. P. Madura, B. Zhang, D. E. Moller, P. Tontonoz, P. Sarraf, B. M. Spiegelman and B. B. Lowell, J. Biol. Chem. 272, 5283 (1997).

26. S. G. Miller, P. De Vos, M. Guerre-Millo, K. Wong, T. Hermann, B. Staels, M. R. Briggs and J. Auwerx, Proc. Natl. Acad. Sci. USA 93, 5507 (1996).

27. R. Saladin, B. Staels, J. Auwerx and M. Briggs, Horm. Metab. Res. 28, 638 (1996).

28. H. Masuzaki, Y. Ogawa, N. Sagawa, K. Hosada, T. Matsumoto, H. Mise, H. Nishimura, Y. Toshimasa, I. Tanaka, T. Mori and K. Nakao, Nature Med. 3,1029 (1997).

29. N. Ghilardi and R. C. Skoda, Mol. Endocrinol. 11, 393 (1997).

30. X. Hu, S. C. Junaje, N. J. Maihle and M. P. Cleary, J. Natl. Cancer Inst. 94,1704 (2002).

31. S. N. O’Brien, B. H. Welter and T. M. Price, Biochem. Biophys Res. Commun. 259, 695 (1999). 